\title{
On Detectability of Switched Linear Differential-Algebraic Equations
}

\author{
Aneel Tanwani
}

\begin{abstract}
This paper addresses the notion of detectability for continuous-time switched systems comprising linear differential-algebraic equations (DAEs). It relates to studying asymptotic stability of the set of state trajectories corresponding to zero input and zero output, with a fixed switching signal. Due to the nature of solutions of switched DAEs, the problem reduces to analyzing stability of the trajectories emanating from a non-vanishing unobservable subspace, for which we first derive a geometric expression. The stability of state trajectories starting from that subspace can then be checked in two possible ways. In the first case, detectability of switched DAE is shown to be equivalent to the asymptotic stability of a reduced order discrete-time switched system. In the second approach, the solutions from a non-vanishing unobservable subspace are mapped to the solutions of a reduced order continuous system with time-varying switching ordinary differential equations (ODEs). As a special case of the later approach, the reduced order switched system is time-invariant if the unobservable subspace is invariant for all subsystems.
\end{abstract}

\section{INTRODUCTION}

We consider a class of systems with switching linear differential-algebraic equations (DAEs) described as:

$$
\begin{aligned}
E_{\sigma} \dot{x} & =A_{\sigma} x+B_{\sigma} u \\
y & =C_{\sigma} x
\end{aligned}
$$

where $x: \mathbb{R} \rightarrow \mathbb{R}^{n}, u: \mathbb{R} \rightarrow \mathbb{R}^{d_{u}}, y: \mathbb{R} \rightarrow \mathbb{R}^{d_{y}}$ denote the state, input and output trajectories of the system respectively. The switching signal $\sigma: \mathbb{R} \rightarrow \mathbb{N}$ is a locally finite, piecewise constant, right-continuous function of time and in our notation it changes its value at time instants $0=t_{0}<t_{1}<t_{2}<\ldots$ called switching times. We adopt the convention that over the interval $\left[t_{k}, t_{k+1}\right)$ of length $\tau_{k}:=t_{k+1}-t_{k}$, the active mode is defined by the quadruple $\left(E_{k}, A_{k}, B_{k}, C_{k}\right), k \in \mathbb{N}$.

Several properties of switched DAEs have been studied in the recent past. This paper is aimed at introducing the notion of detectability, which relates to the question whether the state trajectories of the system converge to the origin when the input $u$, and the observed output $y$ are identically zero. This question, of course, can be addressed in several different ways depending on for what class of switching signals do we seek stability of the dynamics of interest.

In our framework, we consider the switching signal to be fixed and given, and study the stability of a subset of trajectories of (1) obtained with $(u, y) \equiv 0$, for that given switching signal. The starting point for addressing this question appears in our previous works [8], [10], where we find an unobservable subspace (of initial conditions) for a given $\sigma$ which produces zero output with zero input.

The authors are with Department of Mathematics, Technische Universität Kaiserslautern, Germany. Email: \{tanwani,trenn @mathematik.uni-kl.de. This work is sponsored by German Science Foundation DFG grant TR 1223/2-1.
Stephan Trenn

Because of the nature of the solutions of the switched DAEs, certain solutions starting from the unobservable subspace may jump to zero in finite time and if the set of initial conditions for which this happens can be identified from system data, then it is not relevant to consider the asymptotic behavior of such trajectories. For this reason, we reduce the problem of detectability to studying the solution starting from a subset of unobservable subspace which do not vanish, or jump to zero. A systematic procedure for computing such a subspace based on linear-algebraic methods is given. It is then seen that the stability of these trajectories can be addressed by studying the stability of certain reduced order switching ordinary difference/differential equations (ODEs), and hence tools from the theory of stability of discretetime or continuous-time switched ODEs can be invoked to complete the solution to our detectability problem at hand.

With regards to the current literature, this paper aims at bridging certain notions from stability of switched systems and observability results obtained in the algebraic setting. To the best of our knowledge, this particular approach towards detectability has not been adopted. A novel aspect of the system class considered in this paper also lies in the fact that the jump maps are typically singular, and the solutions may involve Dirac impulses and its derivatives in addition to jumps. For switched linear systems without jumps, one finds certain results on detectability [3] which relate to extending the Kalman decomposition to switched systems and define detectability as the stability of the dynamics reduced to a common invariant subspace (similar result would appear as a particular case of our main result in Section VI).

Some recent results on a related notion of output-tostate stability for switched nonlinear systems appear in [6], where the focus is on characterizing a class of switching signals under which the growth of the state trajectory is bounded by some increasing function of the output norm. This approaches typically requires some subsystems to be completely detectable and the stabilizing switching signals are the ones for which the detectable subsystems are active sufficiently longer than non-detectable ones.

In our work, detectability of individual subsystems is not required but this generality comes at the price of working with a fixed switching signal, and knowing the exact switching times to formulate an expression of unobservable subspace, whose stability is then taken into account. For nonswitched DAEs, one can find references related to detectability in [1].

The outline of this paper is as follows: In Section II we collect some preliminary results related to the solutions of switched DAEs, and related stability notions. We formulate the definition of detectability in Section III and reduce the problem to the stability of non-vanishing unobservable trajectories. A geometric expression of the set of initial conditions 
generating such trajectories is presented in Section IV, and their stability criteria is studied in Section $\mathrm{V}$ in terms of certain reduced-order time-varying systems. In Section VI, we treat a special case where the unobservable subspace satisfies certain invariance condition, and the reduced-order subsystems that we obtain are time-invariant whose stability, and hence detectability of the original system, can be checked using the tools from stability of switched ODEs.

\section{PRELIMINARIES}

We recall some algebraic tools which will be used in defining the solution of a switched DAE (1) and also used heavily in deriving conditions for detectability.

\section{A. Properties of a matrix pair $(E, A)$}

We start by collecting important properties and definitions for matrix pairs $(E, A)$. We only consider regular matrix pairs, i.e. for which the polynomial $\operatorname{det}(s E-A)$ is not the zero polynomial. A very useful characterization of regularity is the following well-known result (see e.g. [2]).

Proposition 1 (Regularity and quasi-Weierstraß form): A matrix pair $(E, A) \in \mathbb{R}^{n \times n} \times \mathbb{R}^{n \times n}$ is regular if, and only if, there exist invertible matrices $S, T \in \mathbb{R}^{n \times n}$ such that

$$
(S E T, S A T)=\left(\left[\begin{array}{cc}
I & 0 \\
0 & N
\end{array}\right],\left[\begin{array}{ll}
J & 0 \\
0 & I
\end{array}\right]\right),
$$

where $J \in \mathbb{R}^{n_{1} \times n_{1}}, 0 \leq n_{1} \leq n$, is some matrix and $N \in$ $\mathbb{R}^{n_{2} \times n_{2}}, n_{2}:=n-n_{1}$, is a nilpotent matrix.

One can calculate the matrices $S, T$ by constructing the so called Wong-sequences from the matrices $E, A$, see [2] for details. Based on these transformation matrices we define the following "projectors" [9].

Definition 2 (Consistency, differential and impulse projectors): Consider the regular matrix pair $(E, A)$ with corresponding quasi-Weierstrass form (2). The consistency projector of $(E, A)$ is given by

$$
\Pi_{(E, A)}=T\left[\begin{array}{ll}
I & 0 \\
0 & 0
\end{array}\right] T^{-1},
$$

the differential projector is given by

$$
\Pi_{(E, A)}^{\text {diff }}=T\left[\begin{array}{ll}
I & 0 \\
0 & 0
\end{array}\right] S
$$

and the impulse projector is given by

$$
\Pi_{(E, A)}^{\mathrm{imp}}=T\left[\begin{array}{ll}
0 & 0 \\
0 & I
\end{array}\right] S,
$$

where the block sizes correspond to the ones in (2). $\triangleleft$

Note that only the consistency projector is a projector in the usual sense (i.e. $\Pi_{(E, A)}$ is an idempotent matrix); whereas $\Pi_{(E, A)}^{\text {diff }}$ and $\Pi_{(E, A)}^{\mathrm{imp}}$ are not projectors because, in general, $\Pi_{(E, A)}^{\text {diff }} \Pi_{(E, A)}^{\text {diff }} \neq \Pi_{(E, A)}^{\text {diff }}$ and the same holds for $\Pi_{(E, A)}^{\mathrm{imp}}$. For a system described by the DAE $E \dot{x}=A x$, the fundamental object of interest is the consistency space defined as

$\mathfrak{C}_{(E, A)}:=\left\{x_{0} \in \mathbb{R}^{n} \mid \exists x \in \mathcal{C}^{1}: E \dot{x}=A x \wedge x(0)=x_{0}\right\}$, where $\mathcal{C}^{1}$ is the space of differentiable functions $x: \mathbb{R} \rightarrow$ $\mathbb{R}^{n}$. It then holds that [2], im $\Pi_{(E, A)}=\mathfrak{C}_{(E, A)}$, and for the initial condition $x(0) \in \mathfrak{C}_{(E, A)}$, there exists a unique solution $x \in \mathcal{C}^{1}$ of $E \dot{x}=A x$ that evolves within $\mathfrak{C}_{(E, A)}$. In fact, these differentiable solutions can be described using an ODE using the definition of differential projector.

Lemma 3 ([9, Lem. 3]): Consider the DAE $E \dot{x}=A x$ with regular matrix pair $(E, A)$. Then any solution $x \in \mathcal{C}^{1}$ of $E \dot{x}=A x$ fulfills

$$
\dot{x}=\Pi_{(E, A)}^{\mathrm{diff}} A x=: A^{\mathrm{diff}} x .
$$

In case the initial condition is inconsistent, the basic idea behind constructing the solution is to introduce a jump that maps the initial condition to $\mathfrak{C}_{(E, A)}$, and after this initial time, the solution is propagated in a smooth manner. When the matrix $N$ in (2) is not zero, the derivatives of jumps may appear in the solution, which are formalized by introducing Dirac impulses and its derivatives. For this reason, we consider the space of piecewise-smooth distributions $\mathbb{D}_{\mathrm{pwC}} \mathcal{C}^{\infty}$ from [11] as the solution space, and seek a solution $x \in$ $\left.\left(\mathbb{D}_{\mathrm{pwC}}\right)^{n}\right)^{n}$ to the following initial-trajectory problem (ITP):

$$
\begin{aligned}
x_{(-\infty, 0)} & =x_{(-\infty, 0)}^{0} \\
(E \dot{x})_{[0, \infty)} & =(A x)_{[0, \infty)},
\end{aligned}
$$

where $x^{0} \in\left(\mathbb{D}_{\mathrm{pw}} \mathcal{C}^{\infty}\right)^{n}$ is some initial trajectory, and $f_{\mathcal{I}}$ denotes the restriction of a piecewise-smooth distribution $f$ to an interval $\mathcal{I}$. In [11], it is shown that the ITP (3) has a unique solution for any initial trajectory if, and only if, the matrix pair $(E, A)$ is regular. In particular, the following result concerning the consistency projector holds.

Lemma 4 (Role of consistency projector [11, Thm. 4.2.8]): Consider the ITP (3) with regular matrix pair $(E, A)$ and with arbitrary initial trajectory $x^{0} \in\left(\mathbb{D}_{\mathrm{pw}} \mathcal{C}_{\infty}\right)^{n}$. Then there exists a unique solution $x \in\left(\mathbb{D}_{\mathrm{pw}} \mathcal{C}^{\infty}\right)^{n}$ and

$$
x\left(0^{+}\right)=\Pi_{(E, A)} x\left(0^{-}\right) .
$$

Finally, the role of the impulsive projector becomes clear when expressing the impulsive part, denoted by $x[0]$, of the distributional solution $x$ of the ITP (3).

Lemma 5 ([9, Cor. 5]): Consider the ITP (3) with regular matrix pair $(E, A)$. Let $E^{\mathrm{imp}}:=\prod_{(E, A)}^{\mathrm{imp}} E$ then, for the unique solution $x \in\left(\mathbb{D}_{\mathrm{pwC}} \infty\right)^{n}$,

$$
x[0]=-\sum_{i=0}^{n-2}\left(E^{\mathrm{imp}}\right)^{i+1} x\left(0^{-}\right) \delta_{0}^{(i)},
$$

where $\delta_{0}^{(i)}$ denotes the $i$-th (distributional) derivative of the Dirac-impulse $\delta_{0}$ at $t=0$.

\section{B. Distributional solutions and stability}

When studying switched DAEs of the form $E_{\sigma} \dot{x}=A_{\sigma} x$ the consistency spaces for each pair $\left(E_{k}, A_{k}\right)$ are different in general. Hence, the switch from one mode to another may introduce jumps, and Dirac impulses (and its derivatives) in the solution. This makes the framework of piecewise smooth distributions [11] a natural candidate for studying the solutions of a switched DAE. More formally, consider a homogenous switched DAE

$$
E_{\sigma} \dot{x}=A_{\sigma} x .
$$

Under the assumption that each pair $\left(E_{k}, A_{k}\right)$ is regular, there exists a solution $x \in\left(\mathbb{D}_{\mathrm{pw}} \mathcal{C}^{\infty}\right)^{n}$ uniquely determined 
by the initial value $x\left(0^{-}\right)$, which can be expressed as:

$$
x=x_{\mathbb{D}}^{f}+\sum_{k \in \mathbb{N}} \sum_{i=0}^{n-2} a_{k}^{i} \delta_{t_{k}}^{(i)}
$$

where $x_{\mathbb{D}}^{f}$ denotes the distribution induced by the piecewise smooth function $x^{f}$ and $\delta_{t_{k}}^{(i)}$ denotes the $i$-th derivative of the Dirac impulse with support at switching time $t_{k}$. The coefficients $a_{k}^{i}$ can be inferred from (4). To study the stability of solutions of switched DAE, we introduce the following definition:

Definition 6: The switched DAE (1) is called asymptotically stable if there exist two class $\mathcal{K} \mathcal{L}$ functions $\beta_{c}, \beta_{d}$, such that every solution $x \in\left(\mathbb{D}_{\mathrm{pwC}} \infty\right)^{n}$, expressed as (5), satisfies

$$
\left|x^{f}(t)\right| \leq \beta_{c}\left(\left|x\left(0^{-}\right)\right|, t\right), \quad \forall t \geq 0
$$

and for each switching time $t_{k} \geq 0$

$$
\sum_{i=0}^{n-2}\left|a_{k}^{i}\right| \leq \beta_{d}\left(\left|x\left(0^{-}\right)\right|, t_{k}\right) .
$$

The motivation for this definition comes from the fact that, under mild assumptions on the switching signal ${ }^{1}$, it follows that $x$ converges to the zero distribution as $t \rightarrow \infty$, i.e., for every compactly supported smooth function $\varphi$ and its time shift $\tau_{t}\{\varphi\}(s):=\varphi(s-t)$ it holds that

$$
x\left(\tau_{t}\{\varphi\}\right)=x_{\mathbb{D}}^{f}\left(\tau_{t}\{\varphi\}\right)+\sum_{t_{k} \geq t} \sum_{i=0}^{n-2}(-1)^{i} a_{k}^{i} \varphi^{(i)}\left(t_{k}-t\right)
$$

converges to zero as $t$ tends to infinity. In fact, the next proposition shows that under a mild boundedness assumption on matrices of system (1) (which is satisfied in case of finitely many subsystems) the $\mathcal{K} \mathcal{L}$ estimate (6) on the function part of $x$ already implies (7).

Proposition 7: Consider the switched DAE (1) with corresponding matrices $E_{k}^{\mathrm{imp}}, k \in \mathbb{N}$ (see Lemma 5) and assume that there exists $M>0$ such that $\left\|E_{k}^{\text {imp }}\right\| \leq M$ for all $k \in \mathbb{N}$ and some induced matrix norm $\|\cdot\|$. Then (1) is asymptotically stable if and only if (6) holds.

Proof: The necessity is obvious. To prove sufficiency, it is first observed that for each $k \in \mathbb{N}, 0 \leq i \leq n-2$, we have

$$
\begin{aligned}
&\left|a_{k}^{i}\right| \stackrel{(4)}{=}\left|\left(E_{k}^{\mathrm{imp}}\right)^{i+1} x\left(t_{k}^{-}\right)\right| \leq M^{i+1}\left|x\left(t_{k}^{-}\right)\right| \\
& \stackrel{(6)}{\leq} M^{i+1} \beta_{c}\left(\left|x\left(0^{-}\right)\right|, t_{k}\right) .
\end{aligned}
$$

By choosing

$$
\beta_{d}\left(r, t_{k}\right):=\sum_{i=0}^{n-2} M^{i+1} \beta_{c}\left(r, t_{k}\right)
$$

the inequality (7) holds.

Because of Proposition 7, we will only check that the function part $x^{f}$ of the distributional solution $x$ is converging to zero to deduce stability of the system under consideration (under the silent boundedness assumption on $E_{k}^{\mathrm{imp}}$ ).

\footnotetext{
${ }^{1}$ Roughly speaking, it needs to be assumed that the density of switching times is upper bounded.
}

Remark 8 (Dirac impulses and stability): In contrast to previous works on stability of switched DAE [4], [5] we do not require impulse-freeness of solutions for asymptotic stability. Rather the stability of the impulsive part is formulated as stability of the coefficients associated with the impulsive parts.

\section{Detectability Notion}

To introduce the notion of detectability, we will limit ourselves to the following subset of system trajectories:

$$
\mathcal{N}^{\sigma}:=\left\{x^{0} \in \mathbb{R}^{n} \mid(x, 0,0) \text { solves }(1) \wedge x\left(0^{-}\right)=x^{0}\right\},
$$

where the triplet $(x, u, y)$ denotes signals satisfying (1) for some given switching signal $\sigma$.

Definition 9: The switched DAE (1) is called detectable for a given switching signal $\sigma$, if there exists a class $\mathcal{K} \mathcal{L}$ function $\beta$ such that, for each distributional solution $(x, 0,0)$ of (1) with $x=x_{\mathbb{D}}^{f}+x[\cdot] \in\left(\mathbb{D}_{\mathrm{pwC}} \infty\right)^{n}$ and $x\left(0^{-}\right) \in \mathcal{N}^{\sigma}$, we have

$$
\left|x^{f}(t)\right| \leq \beta\left(\left|x\left(0^{-}\right)\right|, t\right) .
$$

Thus, in our problem formulation, we only consider the stability of output-zeroing dynamics for a fixed switching signal under zero input. The goal of this paper is to develop conditions for checking this property. Note that due to linearity of (1), our detectability definition is equivalent to the property that any two state trajectories corresponding to the same input and output converge towards each other, which will be important for the observer design; however, this is not the topic of this paper and we therefore work with the simplified definition.

Since the systems under consideration are switched DAEs, it is entirely possible that a state trajectory starting with a nonzero initial condition may jump to zero at some time. From stability point of view, it is not relevant to talk about such trajectories and hence we can exclude them from the set $\mathcal{N}^{\sigma}$. More formally, let

$$
\mathcal{N}_{0}^{\sigma}:=\left\{\begin{array}{l|l}
x\left(0^{-}\right) & \begin{array}{l}
(x, 0,0) \text { solves }(1) \wedge \\
\exists t \in[0, \infty) \text { s.t. } x(t)=0
\end{array}
\end{array}\right\} .
$$

Clearly, $\mathcal{N}_{0}^{\sigma} \subseteq \mathcal{N}^{\sigma}$, hence we can choose a nonvanishingunobservable subspace $\overline{\mathcal{N}}_{0}^{\sigma} \subseteq \mathbb{R}^{n}$ such that

$$
\mathcal{N}^{\sigma}=\mathcal{N}_{0}^{\sigma} \oplus \overline{\mathcal{N}}_{0}^{\sigma}
$$

that is, $\overline{\mathcal{N}}_{0}^{\sigma}$ comprises a set of initial conditions which result in zero output with zero input, but the corresponding state trajectories do not jump to zero in finite time. Note that the choice of $\overline{\mathcal{N}}_{0}^{\sigma}$ is not unique. From a detectability viewpoint, we are only concerned about one possible candidate for $\overline{\mathcal{N}}_{0}^{\sigma}$, and derive conditions for asymptotic stability of the state trajectories starting from the set $\overline{\mathcal{N}}_{0}^{\sigma}$. The discussion can be summed up as follows:

Proposition 10: Consider a subspace $\overline{\mathcal{N}}_{0}^{\sigma} \subseteq \mathcal{N}^{\sigma}$ satisfying (11). Then (1) is detectable if, and only if, (9) holds for each solution $(x, 0,0)$ of (1) with $x\left(0^{-}\right) \in \overline{\mathcal{N}}_{0}^{\sigma}$.

In the remainder of this paper, we want to study tools for computing some set $\overline{\mathcal{N}}_{0}^{\sigma}$ (Section IV) and conditions for stability of trajectories starting with initial condition in $\overline{\mathcal{N}}_{0}^{\sigma}$ (Section V). 


\section{Geometric construction of $\overline{\mathcal{N}}_{0}^{\sigma}$}

In this section, we will give a geometric construction of the subspace $\overline{\mathcal{N}}_{0}^{\sigma}$, which intuitively refers to the subspace of initial conditions of nonvanishing-unobservable trajectories. The desired construction requires us to first recall the results on undeterminable ${ }^{2}$ subspaces from our previous work [9], [10].

\section{A. Undeterminable subspaces for switched DAEs}

We define for $k \geq 0$ :

$$
\begin{aligned}
\Pi_{k} & :=\Pi_{\left(E_{k}, A_{k}\right)}, \quad \mathfrak{C}_{k}:=\mathfrak{C}_{\left(E_{k}, A_{k}\right)}, \\
O_{k}^{\text {diff }} & :=\left[C_{k} \Pi_{k} / C_{k} A_{k}^{\text {diff }} / \cdots / C_{k}\left(A_{k}^{\text {diff }}\right)^{n-1}\right], \\
O_{k}^{\text {imp }} & :=\left[C_{k} E_{k}^{\text {imp }} / C_{k}\left(E_{k}^{\text {imp }}\right)^{2} / \cdots / C_{k}\left(E_{k}^{\text {imp }}\right)^{n-1}\right] .
\end{aligned}
$$

In view of Lemma $3, O_{k}^{\text {diff }}$ is the Kalman observability matrix of the ODE system:

$$
\dot{x}=A_{k}^{\text {diff }} x, \quad y=C_{k} x=C_{k} \Pi_{k} x
$$

taking into account that $x$ only evolves within the consistency space (yielding $\Pi_{k} x=x$ ) as well as $\Pi_{k} A_{k}^{\text {diff }}=A_{k}^{\text {diff }}$. Making use of Lemma 5, it is seen that the matrix $O_{k}^{\text {imp }}$ defines the mapping from $x\left(t_{k}^{-}\right)$to $y\left[t_{k}\right]$. As done in [10], these matrices allow us to define the local unobservable space $\mathcal{M}_{k}, k \geq 0$, as follows

$$
\begin{aligned}
& \mathcal{M}_{0}:=\operatorname{ker} O_{0}^{\text {imp }} \cap \operatorname{ker} O_{0}^{\text {diff }} \Pi_{0} \\
& \mathcal{M}_{k}:=\mathfrak{C}_{k-1} \cap \operatorname{ker} O_{k-1}^{\text {diff }} \cap \operatorname{ker} O_{k}^{\text {imp }} \cap \operatorname{ker} O_{k}^{\text {diff }} \Pi_{k}, \quad k \geq 1,
\end{aligned}
$$

so that it is possible to recover $x\left(t_{k}^{-}\right)$modulo $\mathcal{M}_{k}$ by using system data and measurements over the interval $\left(t_{k-1}, t_{k+1}\right)$. We next define the subspaces $\mathcal{Q}^{k}, k \geq 0$, as follows:

$$
\begin{aligned}
\mathcal{Q}^{0} & :=\Pi_{0} \mathcal{M}_{0}, \\
\mathcal{Q}^{k+1} & :=\Pi_{k+1}\left(\mathcal{M}_{k+1} \cap e^{A_{k}^{\text {diff }} \tau_{k}} \mathcal{Q}^{k}\right), k \geq 1 .
\end{aligned}
$$

The intuition behind the definition of the subspace $\mathcal{Q}^{k}$ is to define the set containing $x\left(t_{k}^{+}\right)$when $y_{\left[t_{0}, t_{k}\right]} \equiv 0$. The recursive definition says that the uncertainty in state at time $t_{k}^{+}$is carried forward under the system dynamics and intersected with the locally unobservable subspace $\mathcal{M}_{k+1}$. The resulting subspace is then mapped to $\mathfrak{C}_{k+1}$ to obtain a smaller set $\mathcal{Q}^{k+1}$ containing $x\left(t_{k+1}^{+}\right)$.

Proposition 11 (Undeterminable states [10]): Consider the switched DAE (1) with zero input. Then $\mathcal{Q}^{k}$ for each $k \geq 0$ characterizes the undeterminable space in the following sense:

$$
y \equiv 0 \quad \Leftrightarrow \quad x\left(t_{k}^{+}\right) \in \mathcal{Q}^{k}, \quad \forall k \geq 0 .
$$

Furthermore, there exists $m^{*} \in \mathbb{N}$ such that $\operatorname{dim} \mathcal{Q}^{k}=$ $\operatorname{dim} \mathcal{Q}^{m^{*}}$ for all $k \geq m^{*}$.

\section{B. Nonvanishing-unobservable subspace}

To find an expression for $\overline{\mathcal{N}}_{0}^{\sigma}$, we consider the following sequence of subspaces. For $m \in \mathbb{N}$, let

$$
\mathcal{P}_{m}^{m}=\mathcal{Q}^{m}
$$

\footnotetext{
${ }^{2}$ In contrast to the unobservable subspace, the undeterminable subspace considers the states evaluated at the end of the corresponding interval.
}

and for $k=m-1, \ldots, 0$, choose $\mathcal{P}_{k}^{m} \subseteq \mathcal{Q}^{k}$ such that

$$
\begin{array}{r}
\operatorname{ker} \Pi_{k+1} e^{A_{k}^{\text {diff }} \tau_{k}}+\left(\mathcal{Q}^{k} \cap e^{-A_{k}^{\text {diff }} \tau_{k}}\left(\mathcal{M}_{k+1} \cap \Pi_{k+1}^{-1}\left(\mathcal{P}_{k+1}^{m}\right)\right)\right) \\
=\operatorname{ker} \Pi_{k+1} e^{A_{k}^{\text {diff }} \tau_{k}} \oplus \mathcal{P}_{k}^{m}, \quad(13 \mathrm{~b})
\end{array}
$$

and $\mathcal{P}_{-1}^{m}$ is chosen so that

$$
\operatorname{ker} \Pi_{0}+\left(\mathcal{M}_{0} \cap \Pi_{0}^{-1}\left(\mathcal{P}_{0}^{m}\right)\right)=\operatorname{ker} \Pi_{0} \oplus \mathcal{P}_{-1}^{m} .
$$

We next state the properties of these subspaces $\mathcal{P}_{k}^{m}$ which will be utilized later and in particular how they relate to $\overline{\mathcal{N}}_{0}^{\sigma}$.

Proposition 12: For $k=0, \cdots, m$, the following statements hold for $\mathcal{P}_{k}^{m}$ :

(i) If $x\left(t_{0}^{-}\right) \in \mathcal{P}_{-1}^{m}$, then $x\left(t_{k}^{+}\right) \in \mathcal{P}_{k}^{m}$, and

(ii) $\operatorname{dim} \mathcal{P}_{k}^{m}=\operatorname{dim} \mathcal{Q}^{m}$.

In particular, $\overline{\mathcal{N}}_{0}^{\sigma}=\mathcal{P}_{-1}^{m^{*}}$, for $m^{*}$ given in Proposition $11 . \triangleleft$

In what follows, it is assumed that the integer $m^{*}$ has been computed for the given switching signal $\sigma$, and we will carry forward the following simplified notation:

$$
\mathcal{P}_{k}:= \begin{cases}\mathcal{P}_{k}^{m^{*}}, & -1 \leq k \leq m^{*} \\ \mathcal{Q}^{k}, & k>m^{*}\end{cases}
$$

For every solution $x$ with initial condition $x\left(t_{0}^{-}\right) \in \mathcal{P}_{-1}^{m^{*}}=$ $\overline{\mathcal{N}}_{0}^{\sigma}$, the statements of Propositions 11 and 12 yield

$$
x\left(t_{k}^{+}\right) \in \mathcal{P}_{k}, \quad \forall k \geq 0 .
$$

\section{Stability CONDitions FOR $\overline{\mathcal{N}}_{0}^{\sigma}$}

The basic idea in studying the stability of solutions of the switched DAE starting from $\mathcal{P}_{-1}=\overline{\mathcal{N}}_{0}^{\sigma}$, is to map them to the solutions of a reduced order switched ODE. This is done in two ways: First, in Section V-A, the sought reduced order system is described by discrete-time switched ODEs and secondly, in Section V-B, the reduced order system is described by continuous-time switched ODEs.

\section{A. Analogy with discrete-time switched ODEs}

To study the stability of solutions of the DAE starting from the subspace $\mathcal{P}_{-1}$, we can introduce a reduced order discrete-time switched ODE which matches the solutions of the original system at switching times when the output is restricted to zero.

Theorem 13: Let $P_{k}$ denote a matrix whose columns comprise an orthonormal basis of $\mathcal{P}_{k}$. Then the switched DAE is detectable for $\sigma$ if and only if the following discretetime system is asymptotically stable for that $\sigma$ :

$$
\eta_{k+1}=\Gamma_{k} \eta_{k}
$$

where $\Gamma_{k}:=P_{k+1}^{\top} \Pi_{k+1} e^{A_{k}^{\text {diff }} \tau_{k}} P_{k} \in \mathbb{R}^{m \times m}, m \leq n$.

Proof: We will show that the solution $x$ of the switched DAE (1) with initial condition $x\left(0^{-}\right) \in \overline{\mathcal{N}}_{0}^{\sigma}=\mathcal{P}_{-1}$ matches a solution of the discrete-time system (14) at switching times $t_{k}, k \in \mathbb{N}$, in the sense that

$$
x\left(t_{k}^{+}\right)=P_{k} \eta_{k}
$$

To see this, note that, if $x\left(0^{-}\right) \in \mathcal{P}_{-1}$, then $x\left(0^{+}\right)=$ $\Pi_{0} x\left(0^{-}\right) \in \mathcal{P}_{0}$, and we can write

$$
x\left(0^{+}\right)=P_{0} \eta_{0}
$$


for some $\eta_{0} \in \mathbb{R}^{m}$. Proceeding inductively, if it holds for some $k \in \mathbb{N}$, that $x\left(t_{k}^{+}\right)=P_{k} \eta_{k}$, then

$$
x\left(t_{k+1}^{+}\right)=\Pi_{k+1} e^{A_{k}^{\text {diff }} \tau_{k}} x\left(t_{k}^{+}\right)=\Pi_{k+1} e^{A_{k}^{\text {diff }} \tau_{k}} P_{k} \eta_{k} .
$$

For a solution $\eta$ of (14), it thus holds that

$$
P_{k+1}^{\top} x\left(t_{k+1}^{+}\right)=\eta_{k+1} \text {. }
$$

Since $x\left(t_{k+1}\right) \in \mathcal{P}_{k+1}$ and $P_{k+1}$ comprises an orthonormal basis of $\mathcal{P}_{k+1}$, it follows that

$$
x\left(t_{k+1}^{+}\right)=P_{k+1} \eta_{k+1} .
$$

Because of this relation, we also have, for each $k \geq 0$

$$
\left|x\left(t_{k}^{+}\right)\right|^{2}=\eta_{k}^{\top} P_{k}^{\top} P_{k} \eta_{k}=\left|\eta_{k}\right|^{2},
$$

hence $\left|x\left(t_{k}^{+}\right)\right|=\left|\eta_{k}\right|$. Thus, the system (14) is asymptotically stable if and only if

$$
\left|x\left(t_{k}^{+}\right)\right| \leq \beta_{d}\left(\left|x\left(t_{0}^{-}\right)\right|, t_{k}\right) .
$$

Without restriction of generality we may assume that $t_{k+1}-$ $t_{k} \leq T$ for $T>0$ (because we also allow "switches" to the same mode), hence (15) implies that, for $t \in\left[t_{k}, t_{k+1}\right)$,

$$
\left|x\left(t^{+}\right)\right| \leq e^{\left\|A_{k}^{\text {diff }}\right\| T}\left\|\Pi_{k}\right\| \cdot\left|x\left(t_{k}^{-}\right)\right|,
$$

and the estimate of the form (6) can thus be obtained for each $t \geq 0$.

\section{B. Analogy with continuous-time switched ODEs}

Checking stability using a discrete-time system may be computationally feasible in several cases, such as, when the switching is persistent and the switching times are exactly known. In some cases, when the switching is not persistent, or the dwell time between switches is long so that the exponential of a matrix is difficult to handle numerically for unstable systems, then it is more useful to work with continuous-time reduced order switched systems. In general, due to time-varying nature of the subspaces $\mathcal{P}_{k}$, the reducedorder comparison system that we obtain is also time-varying. In the special case, when the unobservable subspace $\mathcal{Q}^{m^{*}}$ is invariant under the flow of all subsequent modes, we obtain a piecewise-constant reduced order switched ODE (see Section VI). Before proceeding with the description of the comparison system, let us state a useful lemma. The proof of this lemma is omitted due to space limitations but a special case of this result appears as Corollary 16 with proof in Section VI.

Lemma 14: Consider a linear time-invariant system $\dot{x}=$ $A x$ over an interval $[0, \infty)$, and assume that the initial condition $x_{0}:=x(0)$ belongs to an $m$-dimensional subspace $\mathcal{P} \subset \mathbb{R}^{n}$. Let $P \in \mathbb{R}^{n \times m}$ be the matrix whose columns form an orthonormal basis of $\mathcal{P}$. Let $R(t):=\left(P^{\top} e^{A^{\top} t} e^{A t} P\right)^{-1 / 2}$ be the square root of the inverse of a positive definite matrix, let $R^{-1}(t)$ denotes the matrix inverse of $R(t)$, and let $\dot{R}(t)$ denote the element-wise derivative of $R(t)$; then there exists $\eta_{0} \in \mathbb{R}^{m}$ such that the solution of the differential equation

$$
\dot{\eta}(t)=-R^{-1}(t) \dot{R}(t) \eta(t), \quad \eta(0)=P^{\top} x_{0}
$$

has the properties that $\eta(t)=e^{A t} P R(t) x(t)$, and $|\eta(t)|=$ $|x(t)|$. In particular, (16) is forward complete.
For an illustration of Lemma 14, let us consider two examples to see how the reduced-order system can be computed.

Example 1: Consider the dynamical system $\dot{x}=A x$ with $A=\left[\begin{array}{cc}a & 1 \\ -1 & a\end{array}\right]$ for which $e^{A t}=e^{a t}\left[\begin{array}{cc}\cos t & \sin t \\ -\sin t \cos t\end{array}\right]$. If we now take any 1-dimensional subspace that contains $x(0)$ and represent its basis with the unit vector $\operatorname{col}\left(m_{1}, m_{2}\right)$, then due to the particular structure of the exponential matrix, we have $R(t)=e^{-a t}$, so that $\dot{R}(t)=-a e^{-a t}$. The desired scalar differential equation is then given by $\dot{\eta}(t)=a \eta(t)$ which in this case is time-invariant. If the scalar ODE is initialized with $\eta(0)=\left(v_{1} v_{2}\right) x(0)$ then it is easily seen that $\eta(t)$ captures the Euclidean-norm of the state trajectory $x(t)$ at each time instant $t \geq 0$.

Example 2: Consider a second-order system which is not diagonalizable with $A=\left[\begin{array}{ll}a & 1 \\ 0 & a\end{array}\right]$ so that $e^{A t}=e^{a t}\left[\begin{array}{ll}1 & t \\ 0 & 1\end{array}\right]$. Choose a subspace for which the unit vector is given by $\operatorname{col}\left(v_{1}, v_{2}\right)$. One can then compute that, in this case,

$$
R(t)=e^{-a t}\left(1+2 v_{1} v_{2} t+v_{2}^{2} t^{2}\right)^{-1 / 2}
$$

so that the desired scalar differential equation for $\eta$ having the property that $|\eta(t)|=|x(t)|$ is given by

$$
\dot{\eta}(t)=\left(a+\frac{v_{1} v_{2}+v_{2}^{2} t}{1+2 v_{1} v_{2} t+v_{2}^{2} t^{2}}\right) \eta(t) .
$$

Based on the statement of Lemma 14, the solutions of a switched DAE corresponding to nonvanishing-unobservable state trajectories could be mapped to a reduced order timevarying system. Let $P_{k}$ denote the matrix whose columns comprise orthonormal basis of the subspace $\mathcal{P}_{k}$. For $t \in$ $\left[t_{k}, t_{k+1}\right)$, we introduce the notation

$$
\begin{aligned}
M_{k}(t) & :=e^{A_{k}^{\text {diff }}\left(t-t_{k}\right)} P_{k} R_{k}(t) \\
R_{k}(t) & :=\left(P_{k}^{\top} e^{A_{k}^{\text {diff }}\left(t-t_{k}\right)} e^{A_{k}^{\text {diff }}\left(t-t_{k}\right)} P_{k}\right)^{-1 / 2},
\end{aligned}
$$

and consider the following switched ODE with jumps

$$
\begin{aligned}
\dot{\eta}(t) & =-R_{k}(t) \dot{R}_{k}(t) \eta(t), \quad t \in\left(t_{k}, t_{k+1}\right) \\
\eta\left(t_{k}^{+}\right) & =P_{k}^{\top} \Pi_{k} M_{k-1}\left(t_{k}\right) \eta\left(t_{k}^{-}\right)
\end{aligned}
$$

with initial value $\eta\left(t_{0}^{-}\right) \in \mathbb{R}^{m}$.

Theorem 15: The switched DAE is detectable for given $\sigma$ if, and only if, the system (17) is globally asymptotically stable for $\sigma$.

Proof: We will show that there exists a solution $\eta$ to (17) such that $|\eta(t)|=|x(t)|$, for each $t \geq 0$, where $(x, 0,0)$ is the solution to (1) with $x\left(0^{-}\right) \in \overline{\mathcal{N}}_{0}^{\bar{\sigma}}$. Let $\eta$ be the solution of (17) corresponding to the initial condition $\eta\left(t_{0}^{+}\right)=P_{0}^{\top} x\left(t_{0}^{+}\right)$, where $x\left(t_{0}^{+}\right)=\Pi_{0} x\left(t_{0}^{-}\right) \in \mathcal{P}_{0}$. Since the system (17) admits a unique solution, then from Lemma 14, we indeed have that $\eta(t)=M_{0}^{\top}(t) x(t), x(t)=M_{0}(t) \eta(t)$ and hence $|x(t)|=|\eta(t)|$ for each $t \in\left[0, t_{1}\right)$. To show that $|x(t)|=|\eta(t)|$, for all $t \geq 0$, we proceed by induction, and assume that $\eta\left(t_{k}^{+}\right)=P_{k}^{\top} x\left(t_{k}^{+}\right), x\left(t_{k}^{+}\right)=P_{k} \eta\left(t_{k}^{+}\right)$, then due to Lemma 14, $x\left(t_{k+1}^{-}\right)=M_{k}\left(t_{k+1}\right) \eta\left(t_{k+1}^{-}\right)$. At the switching instant $t_{k+1}$, we have

$$
x\left(t_{k+1}^{+}\right)=\Pi_{k+1} x\left(t_{k+1}^{-}\right)=\Pi_{k+1} M_{k}\left(t_{k+1}\right) \eta\left(t_{k+1}^{-}\right)
$$

so that the jump relation in (17b) could be written as:

$$
\eta\left(t_{k+1}\right)=P_{k+1}^{\top} x\left(t_{k+1}\right)=M_{k+1}\left(t_{k+1}\right)^{\top} x\left(t_{k+1}\right) .
$$


The application of Lemma 14 now gives $|x(t)|=|\eta(t)|$ for $t \in\left[t_{k+1}, t_{k+2}\right)$, and hence the desired result follows.

\section{INVARIANT UNDETERMINABLE SUBSPACE}

The statement of Lemma 14 simplifies considerably if the subspace $\mathcal{P}_{k}$ is $A$-invariant, that is, $A \mathcal{P} \subseteq \mathcal{P}$. In particular, the reduced order ODE in this case is time-invariant.

Corollary 16: Consider a linear time-invariant system $\dot{x}=$ $A x$ over an interval $[0, \infty)$, and assume that the initial condition $x_{0}:=x(0)$ belongs to an $m$-dimensional subspace $\mathcal{P} \subset \mathbb{R}^{n}$, which has the property that

$$
A \mathcal{P} \subseteq \mathcal{P}
$$

Let $P \in \mathbb{R}^{n \times m}$ be the matrix whose columns form an orthonormal basis of $\mathcal{P}$. Then $\eta=P^{\top} x$ satisfies the differential equation

$$
\dot{\eta}(t)=P^{\top} A P \eta(t), \quad \eta(0)=P^{\top} x_{0}
$$

and has the property that $|\eta(t)|=|x(t)|$. Furthermore, $\eta(t) \in$ $\mathcal{P}$ for all $t \in[0, \infty)$.

Proof: We let $M:=P$, and $\bar{M}(t):=e^{-A^{\top} t} \bar{P} \in$ $\mathbb{R}^{n \times(n-m)}$, where $\bar{P}$ is an orthonormal matrix such that $\bar{P}^{\top} P=0$. It then follows that $P^{\top} P=I_{m \times m}$, and

$$
\bar{M}^{\top}(t) M=\bar{P}^{\top} e^{-A t} P=0_{(n-m) \times m} .
$$

where the last inequality follows because $e^{-A t} P \in \mathcal{P}$. Thus, $\left(\begin{array}{c}\eta(t) \\ 0\end{array}\right)=\left[\begin{array}{c}M^{\top} \\ M^{\top}(t)\end{array}\right] x(t)$. Since $M$ is orthonormal, and $\bar{M}(t)$ is orthogonal to $M$ for each $t$, we have $x=M \eta=P \eta$. The differential equation for $\eta$ now follows by differentiating both sides of $\eta=P^{\top} x$ with respect to time.

\section{A. Invariant Undeterminable Subspace and Switched ODEs}

Proposition 17: If $\mathcal{Q}^{m^{*}}$ given in Theorem 11 is such that

$$
A_{k}^{\text {diff }} \mathcal{Q}^{m^{*}} \subseteq \mathcal{Q}^{m^{*}} \subseteq \mathcal{M}_{k}, \quad \forall k \in \mathbb{N}
$$

then the switched system (1) is detectable if and only if the following switched ODE with jumps is asymptotically stable:

$$
\begin{aligned}
\dot{\eta}(t) & =P^{\top} A_{k} P \eta(t) \\
\eta\left(t_{k}^{+}\right) & =P^{\top} \Pi_{k+1} P \eta\left(t_{k}^{-}\right)
\end{aligned}
$$

where $P$ is a matrix whose columns comprise an orthonormal basis of $\mathcal{Q}^{m^{*}}$.

The proof is not included here due to space constraints, but we would like to highlight, that the key observation is that (19) implies

$$
\mathcal{P}_{k}=\mathcal{Q}^{m^{*}} \quad \forall k \in \mathbb{N} .
$$

Note that $A_{k}^{\text {diff }}$-invariance of all local unobservable spaces $\mathcal{M}_{k}$ is neither necessary nor sufficient for (19).

The result of Proposition 17 is particularly useful where one can compute an invariant unobservable subspace. Due to invariance, the computation of such unobservable subspace would not depend on the switching times.

\section{B. Special Case: Switched ODEs}

In case of switched ODEs with nonsingular jumps, one can compute this invariant unobservable subspace directly from the system data and it can be shown that any switching signal with a periodic mode sequence would result in this unobservable subspace for almost all switching times [7]. Computing such invariant subspaces for switched DAEs is a topic of ongoing work.

To be specific, we compute $\mathcal{Q}^{m *}$ for switched ODEs without jumps, we assume that the set $\mathcal{S}$, which determines all possible subsystems of system (1) is of the form $\{0,1, \ldots, p\}$, and that the switching signal $\sigma$ has a periodic mode sequence:

$$
\sigma\left(t_{k}\right)=k \bmod (\mathrm{p}+1)
$$

In the description of system (1), we now let $E_{k}$ to be the identity matrix for each $k \in \mathbb{N}$. Using the notation $\langle\mathcal{V} \mid A\rangle$ to denote the largest $A$-invariant subspace contained in the subspace $\mathcal{V}$, one now introduces the sequence of following subspaces:

$$
\begin{aligned}
\mathcal{V}_{0} & :=\left\langle\operatorname{ker} C_{0} \mid A_{0}\right\rangle \cap\left\langle\operatorname{ker} C_{1} \mid A_{1}\right\rangle \cap \ldots\left\langle\operatorname{ker} C_{p} \mid A_{p}\right\rangle \\
\mathcal{V}_{i+1} & :=\left\langle\mathcal{V}_{i} \mid A_{0}\right\rangle \cap\left\langle\mathcal{V}_{i} \mid A_{1}\right\rangle \cap \ldots\left\langle\mathcal{V}_{i} \mid A_{p}\right\rangle .
\end{aligned}
$$

Clearly, we have the inclusions $\mathcal{V}_{0} \supseteq \mathcal{V}_{1} \supseteq \ldots$ and due to finite-dimensionality of the state space, there exists a minimal element of the sequence which we denote by $\mathcal{V}^{*}$. It is shown in [7] that $\mathcal{V}^{*}$ is the unobservable subspace corresponding to a switching signal of the form (21) for almost all switching times. Clearly, $\mathcal{V}^{*}$ satisfies the invariance condition listed in (19) by construction with $A_{k}^{\text {diff }}=A_{k}$. Hence, in this case the problem of detectability reduces to checking the stability of the following reduced-order switched ODE:

$$
\dot{\eta}(t)=P^{\top} A_{k} P \eta(t)
$$

where $P$ now denotes the matrix whose columns comprise orthonormal basis of the subspace $\mathcal{V}^{*}$.

\section{REFERENCES}

[1] F.J. Bejarano, T. Floquet, W. Perruquetti, and G. Zheng. Observability and detectability of singular linear systems with unknown inputs. Automatica, 49(3):793 - 800, 2013.

[2] T. Berger, A. Ilchmann, and S. Trenn. The quasi-Weierstraß form for regular matrix pencils. Lin. Alg. Appl., 436(10):4052-4069, 2012.

[3] E. De Santis, M.D. Di Benedetto, and G. Pola. On observability and detectability of continuous-time linear switching systems. In Proc. 42nd IEEE Conf. Decision \& Control, pages 5777-5782, 2003.

[4] D. Liberzon and S. Trenn. On stability of linear switched differential algebraic equations. In Proc. IEEE 48th Conf. on Decision and Control, pages 2156-2161, December 2009.

[5] D. Liberzon and S. Trenn. Switched nonlinear differential algebraic equations: Solution theory, Lyapunov functions, and stability. Automatica, 48(5):954-963, May 2012.

[6] M.A. Muller and D. Liberzon. Input/output-to-state stability and state-norm estimators for switched nonlinear systems. Automatica, 48(9):2029-2039, 2012.

[7] Z. Sun, S.S. Ge, and T. H. Lee. Controllability and reachability criteria for switched linear systems. Automatica, 38:775-786, 2002.

[8] A. Tanwani, H. Shim, and D. Liberzon. Observability for switched linear systems: Characterization and observer design. IEEE Trans. Autom. Control, 58(4):891-904, 2013.

[9] A. Tanwani and S. Trenn. On observability of switched differentialalgebraic equations. In Proc. 49th IEEE Conf. Decis. Control, Atlanta, USA, pages 5656-5661, 2010.

[10] A. Tanwani and S. Trenn. Observability of switched differentialalgebraic equations for general switching signals. In Proc. 51st IEEE Conf. Decis. Control, Maui, USA, pages 2648-2653, 2012.

[11] S. Trenn. Distributional differential algebraic equations. $\mathrm{PhD}$ thesis, Institut für Mathematik, Technische Universität Ilmenau, Universitätsverlag Ilmenau, Ilmenau, Germany, 2009. 